\title{
Virtual Sink Rotation: Low-Energy Scalable Routing Protocol for Ubiquitous Sensor Networks*
}

\author{
Lynn Choi ${ }^{1}$, Kwangseok Choi ${ }^{1}$, Jungsun $\mathrm{Kim}^{2}$, and Byung Joon Park ${ }^{3}$ \\ ${ }^{1}$ Korea University, Anam-Dong, Sungbuk-Ku, Seoul, Korea \\ \{lchoi, jsheo, hyogon\} akorea.ac.kr \\ Tel: +82-2-3290-3249, Fax: +82-2-921-0544 \\ ${ }^{2}$ School of Electrical Engineering and Computer Science, Hanyang University \\ jskimecse.hanyang.ac.kr \\ ${ }^{3}$ Department of Computer Science, Kwangwoon University \\ bjpark@cs. kwangwoon.ac.kr
}

\begin{abstract}
In this paper we propose a new routing protocol called virtual sink rotation (VSR) routing for large-scale sensor networks. VSR can efficiently handle a large number of sources as well as a large number of sinks with potential mobility. Each sensor node is not required to know the global network topology nor the location awareness. The main ideas underlying the VSR are two folds. First, to alleviate the frequent location updates associated with multiple mobile sinks, the algorithm introduces a virtual sink, which acts as a data collection and dissemination center to collect the data from all the sources and forward them to the actual sinks. This virtual sink can easily support multiple sinks as well as the mobility of the sinks. Second, to address the excessive energy consumption among the sensor nodes around a sink, VSR employs virtual sink rotation, which distributes the role of the virtual sink over all the participating sensor nodes, thus achieving a uniform energy distribution across the entire sensor field and prolonging the lifetime of the network. Experimentation results confirm that the VSR routing can significantly save energy while it can also reduce both the message delay and the message delivery failures compared to previous schemes.
\end{abstract}

\section{Introduction}

Sensor network routing protocols for stationary sinks has been well studied [1, 2, $3,6,7,8,10,11,13]$. However, the routing protocols for mobile sinks still remain an open area, where no practical solution has been discovered. Sink mobility brings new challenges to large-scale sensor networking. First, the location information of a mobile sink needs to be continuously propagated throughout the sensor field to keep all sensor nodes updated with the location to send future data reports. Unfortunately the frequent location updates from a mobile sink can lead

* This research was supported by University IT Research Center Project under contract number C1090-0401-0014. 
to excessive drain of sensors' limited battery power supply and increased collisions in wireless transmissions. Second, the location of a mobile sink can be lost since often the location update may not be fast enough. In such cases a message sent from a source may not be delivered to the mobile sink. Third, the preconstruction of message delivery network or tree cannot be performed since the sink may move and the existing tree may not help immediately after the movement. Fourth, this situation becomes exacerbated when the number of such mobile sinks grows.

Although several sensor network routing protocols have been proposed recently to target mobile sinks, such as TTDD [11], SEAD [7], and HLETDR [2], most of them suggest that each mobile sink needs to continuously propagate its location information throughout the sensor field, either by a local flooding guided by geographical grids pre-maintained [11] or by a global flooding based on localized interactions among the neighboring nodes through interest propagation and reinforcement [2, 6]. Moreover, some of existing schemes assume location awareness using GPS-enabled nodes [7, 11]. However, GPS may not be used in many wireless sensor networks as GPS can work only outdoors with no obstruction and the cost of GPS receivers prevents them from using in low-cost sensor nodes. Furthermore, most of the proposed localization techniques today depend on recursive trilateration/multilateration techniques, which would not provide enough accuracy in wireless sensor networks [4]. Thus, none of the existing approaches provides an efficient and practical solution to this problem.

In this paper we propose a new routing protocol called virtual sink rotation (VSR) routing for large-scale sensor networks. VSR can efficiently handle a large number of sources as well as a large number of sinks with potential mobility. Each sensor node is assumed to be GPS-free, thus suitable for low-cost sensor node implementation. In addition, each node maintains information about its neighbors, thus no global network topology information needs to be maintained. This enables to build low-cost scalable routing protocols for large-scale sensor networks.

The main ideas underlying the VSR are two folds. First, to alleviate the frequent location updates associated with multiple mobile sinks, the algorithm introduces a virtual sink, which acts as a data collection and dissemination center for all the sensor nodes. The virtual sink builds a spanning tree encompassing all the sensor nodes in the field to collect and sometimes to aggregate the data from all the sources. Then, the virtual sink builds a multi-cast tree to the actual sinks to disseminate the data collected from the sources. This virtual sink can easily support multiple sinks as well as the mobility of the sinks since each sensor node does not need to keep track of sink's location information any more. Only the virtual sink needs to keep track of mobile sinks' locations. Second, to address the excessive energy consumption in the sensor field around a sink, VSR employs virtual sink rotation, which distributes the role of the virtual sink over all the sensor nodes participating. This enables a uniform distribution of energy consumption across the entire sensor field so that the lifetime of the sensor network can be extended. Thus, the excessive energy drain of a particular area does neither cause network partitioning nor it creates the holes in the sensor field coverage. Experimentation results confirm that the VSR routing can significantly save energy while it can also reduce both the message delay and the message delivery failures compared to previous schemes. 


\section{Related Works}

While most of existing routing protocols implicitly assume sensor networks with stationary sinks or sinks with very low mobility, TTDD [11] and SEAD [7] specifically target sensor networks with multiple mobile sinks. In TTDD each data source proactively builds a grid structure, which enables mobile sinks to continuously receive data on the move by flooding queries within a local cell only. TTDD's design exploits the fact that sensor nodes are stationary and locationaware to construct and maintain the grid structures with low overhead. A more recent scheme called SEAD resembles VSR in the sense that SEAD does not rely on local or global flooding. Instead, SEAD builds an overlay multicast network called d-tree from each source to multiple sinks. To minimize data dissemination cost, source data is replicated at selected nodes between the source and the sinks. SEAD is different from VSR in that each sensor node in SEAD is assumed to be aware of its geographical location. In addition, the d-tree in SEAD is not a spanning tree but an overlay network connecting a single source and multiple sinks on top of underlying location-based routing protocols such as simple geographical forwarding $[10,13]$.

The rest of this paper is organized as follows. In Section 3 we present the concepts of VSR routing, namely the virtual sink and virtual sink rotation, and describes its relevant algorithms required for its spanning tree construction, virtual path setup and removal, and virtual sink selection policies. In Section 4 we present our simulation methodology and the evaluation results of VSR compared to existing schemes. Section 5 concludes the paper and describes our future work.

\section{Virtual Sink Rotation (VSR) Routing}

\subsection{Virtual Sink}

Virtual Sink Rotation (VSR) routing algorithm is based on two important concepts: virtual sink and virtual sink rotation. In this section we first describe the concept of virtual sink and its associated algorithms.

Virtual Sink. A virtual sink is a sensor node that is delegated to perform the role of all the actual sinks. A sensor node selected as a virtual sink is authorized to act as a data collection center for all the sources and also as a data dissemination center for all the sinks. To perform the data collection and dissemination efficiently, the virtual sink builds a spanning tree called VS tree that encompasses all the sensor nodes in the field to collect and potentially aggregate the data from all the sources. The VS tree is constructed at the initial deployment of sensor nodes and can be repaired or reconstructed from time to time due to node failures, excessive energy drains surrounding the virtual sink, or the additional deployment of new sensor nodes. Once the VS tree is constructed, the virtual sink can act as a data dissemination center for sinks. To forward the data sent from a source, the virtual sink builds a data dissemination path from the source to the actual sink through the virtual sink. When multiple sinks are present, multiple data dissemination paths from the virtual sink to the actual sinks can naturally form a dissemination multi-cast tree to disseminate the data collected from the 
sources to the actual sinks. This virtual sink can easily support multiple sinks as well as the mobility of the sinks since each sensor node does not need to keep track of sink's location information anymore. Only the virtual sink needs to keep track of mobile sinks' locations. And, the location update from an actual sink to the virtual sink can be easily performed using the existing path in the VS tree without local or global forwarding required by previous schemes $[2,6,11]$.

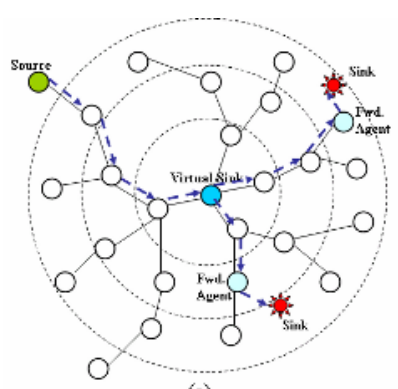

(a)

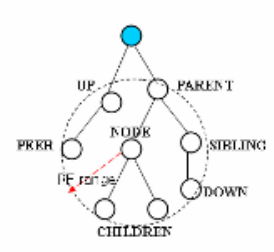

(b)

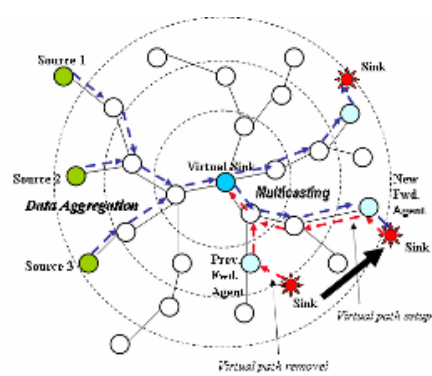

(c)

Fig. 1. (a) An example of VS tree consisting of 26 sensor nodes organized into 4 layers, (b) classification of neighbor nodes, (c) an illustration of a virtual path removal and a new virtual path setup on sink movement

VS Tree Construction. VSR organizes the sensor network into layers, where nodes that belong to a layer have the same hop-count to the virtual sink. Thus, layer 1 consists of nodes which can reach the virtual sink in one hop; layer 2 nodes reach the virtual sink in two hops, and so on. Figure 1(a) illustrates how 26 sensor nodes are organized into four layers. Thus, all the sensor nodes in the sensor field constitute a spanning tree rooted at the virtual sink. The VS tree construction is initiated by a tree setup message sent by the virtual sink. This tree setup message contains its own id, its parent node id, and the number of hops to the virtual sink. All the nodes hearing the message from the virtual sink become the children of the virtual sink and constitute the layer 1 nodes. After hearing this message, nodes in the layer 1 rebroadcast the tree setup message to reach the layer 2 nodes. By overhearing these tree setup messages from a layer below, the virtual sink can record its children nodes that it can reach in a single hop. This process is repeated until all the sensor nodes can be reached by the tree setup message. Using the broadcast nature of wireless transmissions, the VS tree can be easily constructed from the root downward and each node is required to send the tree setup message only once. To reduce the communication latency between a sensor node and the virtual sink, the height of this tree must be minimized since the height of a node represents the number of hops to reach the virtual sink. And, the result tree must span all the sensor nodes in the field. In this regard, the VS tree construction algorithm is to build a spanning tree with a minimum height.

In a VS tree neighbors of a node can be classified as either UP, DOWN, or PEER depending on their proximity to the virtual sink. A node $i$ is considered as UP with respect to a node $j$ if $H(i)<H(j)$, where $H(i)$ denotes the height of a node $i$. Similarly a node $i$ is considered DOWN with respect to a node $j$ if $H(i)>H(j)$. Finally a node $i$ is considered PEER with respect to node $j$ if $H(i)=H(j)$. Each node should have at least 
one UP node in its neighbors to disseminate its data to the virtual sink. Each node selects one in UP neighbors as a PARENT node and it becomes the CHILD node of the PARENT. In addition, a node may or may not have DOWN neighbors depending on its location in the VS tree. To forward the data sent from the virtual sink to an actual sink, each node in this path needs to forward the data coming from its UP neighbor to one of its DOWN nodes. Thus, both UP and DOWN links need to be maintained for the data dissemination. In summary, each node has at least one PARENT node and zero or more CHILDREN nodes. And, its all other neighboring nodes are classified into UP, DOWN, and PEER as illustrated in Figure 1(b).

Virtual Path. Once the VS tree is constructed, the virtual sink can function as a data collection center for all the sources in the field. Each sensor node has a path to the virtual sink through its PARENT node upward in the VS tree and this upward path is called its report path. Now to disseminate the data sent from the sources, the virtual sink needs to build a path downward to real sinks. This downward path is called virtual path. The upward path in connection with the downward path constitutes the data dissemination path from a source to an actual sink. To establish the virtual path to each real sink, the virtual sink must be informed of the location of a real sink. This process is called virtual path setup and initiated by each real sink. Since every sensor node in VS tree already knows the path to the virtual sink, the virtual path setup can be easily performed in VSR using the existing report path.

Virtual Path Setup and Removal. In VSR, each mobile sink is associated with one stationary sensor node called forwarding agent. When a new sink enters the sensor field, the sink selects one of its neighboring sensor nodes that has the smallest hop count to the virtual sink and sends the virtual path setup message to the neighbor node. The selected sensor node is called the sink's forwarding agent. Since this forwarding agent already knows its report path to the virtual sink, the virtual path can be easily set up using this existing path from the agent to the virtual sink. Since the direction of data movement in the virtual path, i.e. downward from the virtual sink to the real sink, is the opposite of the report path, this setup message is forwarded upward to the virtual sink through the report path of the forwarding agent. On its way upward, each node in the virtual path records the direction of a new sink. When the virtual path setup message reaches the virtual sink, the virtual sink can now record the direction of the new sink. Finally, the virtual sink can function as a data dissemination center for the new sink. This way the data dissemination path from all the sources to the particular sink can be established. The virtual path setup is also performed when a sink moves or when the VS tree is reconstructed.

When a sink is about to move out of the range of its current forwarding agent, the existing virtual path to the sink is no longer valid. During this sink movement, the data sent from sources can be lost if the setup of a new virtual path is not fast enough. To avoid such message delivery failures, VSR employs virtual path removal. That is, the mobile sink informs its forwarding agent of its movement and lets its agent to invalidate the old virtual path by sending virtual path removal message. This message must be propagated to the virtual sink through the old virtual path. If a node in the old virtual path already has the data to send to the mobile sink, this data can be sent back to the virtual sink that can temporarily buffer all the messages toward the mobile sink. 
Once the new location of the mobile sink is stabilized, the sink must set up the virtual path again by selecting one of its neighbors as a new forwarding agent and sending the virtual path setup message to this agent. When this message reaches the virtual sink, the virtual sink can now forward all the data to the mobile sink including the buffered messages during the mobile sink's movement. Using the virtual path setup and removal, VSR can successfully forward messages for the mobile sink without losing messages and without indirect forwarding between the agents [11]. As we demonstrate in Section 3, this virtual path setup can be performed very efficiently in terms of energy, delay, and the rate of success deliveries.

Dissemination Multicast Tree. When the number of sinks grows, virtual paths from multiple sinks can be naturally grouped into a multi-cast tree without any additional tree construction overhead. This is because every virtual path is already embedded in the VS tree. When two virtual paths join at a node in the VS tree, two virtual paths can be naturally combined from the join node upward to the virtual sink. The result tree starts from the virtual sink and ends at the two mobile sinks. When the number of sinks grows, the tree naturally expands. The root of the tree is the virtual sink whereas the leaves of the tree are the mobile sinks. We call this tree dissemination multicast tree, which can be used to multicast data more efficiently. Since each virtual path is embedded in the VS tree, the result multi-cast tree can easily exploit the spatial locality among the mobile sinks by utilizing the path proximity among the virtual paths. Both the virtual path and the dissemination multi-cast tree are embedded in the VS tree as illustrated in Figure 1(c). With the dissemination multicast tree, the virtual sink can now act as a data dissemination center for all the sinks in the field.

Note that all the communication in VSR is strictly local, i.e. neighbor-to-neighbor. That is to say, every sensor node including the virtual sink needs to keep track of only its neighbors. This suggests that VSR has two desirable features. First, a sensor node in VSR is not assumed to be aware of its location, i.e. does not rely on GPS to implement the routing protocol. Thus, VSR can be easily employed in low-cost GPS-free sensor node implementations. Second, a sensor node does not have to know the global topology of the network, which implies that each node only keeps track of information about neighbor nodes in its routing table. Thus, the VSR is scalable in terms of the number of nodes in the network since the amount of information kept in each node is constant even though the number of nodes in the field grows.

\subsection{Virtual Sink Rotation}

One of the key issues in low-energy sensor network protocol design is the excessive energy drain among the nodes around a sink. Since a sink usually communicates with a number of sources, this type of many-to-one communication causes heavy traffic around the sink. Thus, interior nodes nearby the sink usually experience more energy consumption than exterior nodes in the field. The same phenomenon occurs among the nodes around the virtual sink in VSR. The situation can be even worse since VSR assumes a normal sensor node as the virtual sink whereas existing routing protocols assume a more powerful sink, which is different from a normal sensor node. To get around this problem, many energy-aware routing protocols have been proposed to distribute the energy consumption throughout the sensor field [3, 8]. However, all of them so far suggest local optimization for this problem in the sense that the sink and 
its immediate neighbors cannot avoid excessive energy drain. Instead, we take a global optimization approach called virtual sink rotation. In virtual sink rotation, when the energy level of the virtual sink reaches a certain threshold, VSR selects another node as a new virtual sink to evenly distribute the energy consumption throughout the sensor field.

Virtual Sink Selection Strategy. If there is only one sink in the field, one of the sink's neighbors can be selected as the virtual sink. When a new sink joins the sensor field, the location of the virtual sink may be far away from this sink depending on its relative position to the previous sink. To minimize energy, the location of the virtual sink must be chosen to minimize the overall communication distance between the virtual sink and multiple sinks. Thus, the optimal position of the virtual sink may be the location corresponding to the weighted center of all the sinks' locations assuming that the sources are evenly distributed throughout the sensor field. However, since each sink is free to move anytime in our network model, we do not take this approach. Instead, we employ global optimization technique called virtual sink rotation, where the role of the virtual sink is rotated among the sensor nodes to distribute the energy as evenly as possible. For the virtual sink selection, we take a simple approach called random selection, which randomly picks one sensor node as the virtual sink. We expect this strategy to perform well compared to a more sophisticated approach assuming that the number of sensor nodes in the field is sufficiently large.

\section{Experimentation and Results}

We implemented the VSR protocol in the ns-2 simulator [9]. The ns-2 simulator implements $1.6 \mathrm{Mbps} 802.11 \mathrm{MAC}$ layer. This MAC layer may not be suitable for sensor networks since it is possible to put the radio in standbye mode during idle intervals as in other sensor network MAC protocols [5, 12]. By contrast, an 802.11 radio consumes as much power when it is idle as when it receives transmissions. To more closely model realistic sensor network model, we altered the ns-2 radio energy model such that the idle-time power dissipation is about $35 \mathrm{~mW}$, reception power dissipation of $395 \mathrm{~mW}$, and transmission power dissipation of $660 \mathrm{~mW}$. This is consistent with previous sensor network studies we compared.

All the simulations data are collected by varying the speed of each mobile sink from 0 to $20 \mathrm{~m} / \mathrm{s}$ in the sensor field of 400 nodes, where the nodes are randomly placed in a $2000 \mathrm{~m} \times 2000 \mathrm{~m}$ square. Five sources and four mobile sinks are used in each simulation run. Each node has a radio range of $250 \mathrm{~m}$. Unless otherwise mentioned, all sources are randomly selected from the sensor field following the random sources model [6] while sinks are uniformly scattered across the field. Each source generates one event per second and each event is modeled as a 64-byte packet. All the events are reported to all the sinks in the field. Each simulation run lasts for $100 \mathrm{sec}$ onds. All the metrics of VSR are compared against only Two-Tier Data Dissemination (TTDD) scheme, which is a protocol specifically designed to handle multiple mobile sinks. 


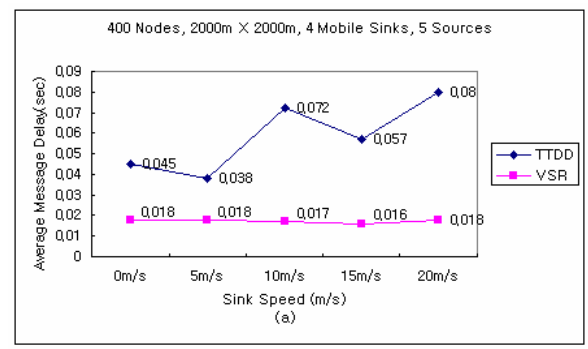

Fig. 2. Average message delay for different speeds of mobile sinks

Figure 2 shows the average message delay for different speeds of mobile sinks. As the speed of mobile sinks increases, VSR's average message delay remains stable. This is because VSR only incurs one virtual path setup for each mobile sink regardless of its speed. By contrast, TTDD requires the former primary agent to forward all the messages to the new immediate agent during the sink movement, which increases the message delay. Moreover, TTDD often results in local flooding to select a new primary agent if the sink moves out of a certain distance from its primary agent. This local flooding increases the contention in the network, further delaying the message delivery during the fast movement of the sinks. However, in VSR the average message delay remains almost the same regardless of the sink speed. Furthermore, the average message delay remains below $20 \mathrm{~ms}$, which is at least twice faster than TTDD for all the cases simulated.

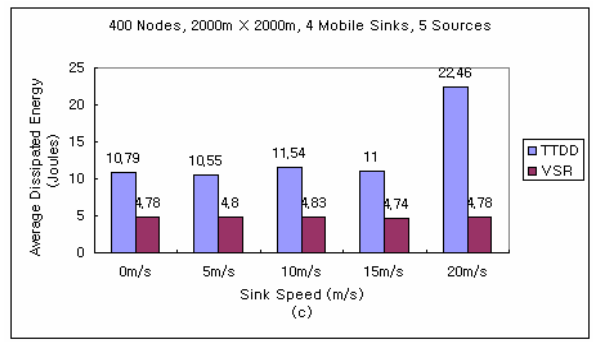

Fig. 3. Average dissipated energy for different speeds of mobile sinks

Figure 3 shows the average dissipated energy. For all different speeds of mobile sinks VSR's average dissipated energy remains relatively constant, around 5J. Similarly, the average dissipated energy of TTDD remains stable at $11 \mathrm{~J}$ for low to medium speed sink movement. However, TTDD's per-node energy consumption jumps to $22.4 \mathrm{~J}$ when the speed of mobile sink reaches $20 \mathrm{~m} / \mathrm{s}$ due to its excessive local flooding and trajectory forwarding caused by the fast movement. Thus, in terms of both energy and delay, VSR substantially outperforms TTDD. 


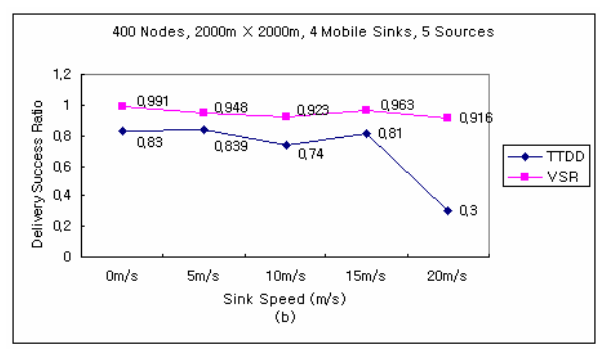

Fig. 4. Delivery success ratio for different speeds of mobile sinks

Lastly, Figure 4 shows the delivery success ratio. As we expect from low delay and low energy consumption of VSR, VSR consistently delivers more than $91 \%$ of all the events to the mobile sinks for all different speeds, whereas TTDD's delivery success ratio remains around $80 \%$ when the speed of mobile sinks ranges from $5 \mathrm{~m} / \mathrm{s}$ to $15 \mathrm{~m} / \mathrm{s}$. However, the TTDD's delivery success ratio suddenly drops to $30 \%$ when the speed of mobile sinks reaches $20 \mathrm{~m} / \mathrm{s}$, which suggests that TTDD's protocol overhead cannot sustain fast movement of mobile sink beyond this speed.

\section{Conclusion}

This paper addresses the problem of scalable and efficient data dissemination in a large-scale sensor network from multiple sources to multiple, potentially mobile sinks. Although routing protocols for sensor networks have been an active research field in the past few years, most of them target sensor networks with immobile sinks or sinks with low mobility. Sink mobility brings new challenges to sensor networking since frequent location updates from mobile sinks often result in unnecessary traffic, which increases the message delay as well as the energy dissipation per node.

In this paper we propose a new routing protocol called virtual sink rotation (VSR) routing for sensor networks. VSR introduces a virtual sink, which acts as a data collection and dissemination center for all the sensor nodes in the field. In addition, VSR employs virtual sink rotation, which distributes the role of the virtual sink over all the participating sensor nodes, thus distributing the energy consumption across the entire sensor field. We have implemented VSR in ns-2 and evaluated the performance of VSR by comparing it to two previous schemes in terms of average message delay, average dissipated energy, and delivery success ratio. Experimentation results confirm that VSR can significantly save energy while it can also reduce both the message delay and the message delivery failures compared to previous schemes. Moreover, each sensor node in VSR is not assumed to know the global network topology nor its position, suggesting that VSR can be a scalable routing solution for large-scale sensor networks implemented with low-cost sensor nodes and mobile sinks.

In our future work, we will investigate several new issues including the impact of data and query aggregation, caching, and the multicasting on the VSR framework. 


\section{References}

1. Al-Karaki, J. N. and Kamal, A. E., Routing Techniques in Wireless Sensor Networks: A Survey, Wireless Communications, IEEE, pp. 6-28, Vol. 11, Issue: 6, Dec. 2004.

2. Baruah, P., Urgaonkar, R., and Krishnamachari, B., Learning-Enforced Time Domain Routing to Mobile Sinks in Wireless Sensor Fields, Local Computer Networks, pp. 525 532, 2004.

3. Boukerche, A., Cheng, X., Linus, J., Energy-aware Data-centric Routing in Microsensor Networks, In Proceedings of the $6^{\text {th }}$ International Workshop on Modeling Analysis and Simulation of Wireless and Mobile Systems, pp. 42 - 49, Sep. 2003.

4. Bulusu, N., Estrin, D., Girod, L. and Heidemann, J., Scalable Coordination for Wireless Sensor Networks: Self-Configuring Localization Systems", In Proceedings of the Sixth International Symposium on Communication Theory and Applications (ISCTA 2001), July 2001.

5. Christian C. Enz., Amre, E. H., Decotignie, J. D., Peiris, V., WiseNET: An Ultralow-Power Wireless Sensor Network Solution, IEEE computer magazine, Vol. 37, No. 8, Aug 2004.

6. Intanagonwiwat, C., Govindan, R. and Estrin, D., Directed Diffusion: A Scalable and Robust Communication Paradigm for Sensor Networks, In Proceedings of the Sixth Annual International Conference on Mobile Computing and Networks, pp. 2 - 16, Vol. 11, Issue 1, 2000.

7. Kim, H. S., Abdelzahan, T. F., Kwon, W. H., Minimum-Energy Asynchronous Dissemination to Mobile Sinks in Wireless Sensor Networks, In Proceedings of the First International Conference on Embedded Networked Sensor Systems, pp. 193 - 204, Nov. 2003.

8. Lindsey, S. and Raghavendra, C. S., Pegasis : Power-Efficient Gathering in Sensor Information Systems, In Proceedings of IEEE international Conference on Communications, pp. $1125-1130$, Vol.3, June 2001.

9. The Network Simulator ns-2 Documentation, http://www.isi.edu/nsman/ns.

10. Xu, Y., Heidemann, J., Estrin, D., Geography-Informed Energy Conservation for Ad Hoc Routing, In Proceedings of the 7th Annual International Conference on Mobile Computing and Networking, pp. $70-84,2001$.

11. Ye, F., Luo, H., Cheng J., Lu, S., Zhang, L., A Two-Tier Data Dissemination Model for Large-Scale Wireless Sensor Networks, In Proceeding of Mobile Computing and Networks, pp. 148 - 159, Sep. 2002.

12. Ye, W., Heidemann, J., Estrin, D., An Energy-Efficient MAC Protocol for Wireless Sensor Networks, In Proceedings of the INFOCOM 2002, pp. 1567 - 1576, Vol. 3, 2002.

13. Yu, Y., Govindan, R. and Estrin, D., Geographical and Energy Aware Routing: A Recursive Data Dissemination Protocol for Wireless Sensor Networks, UCLA Computer Science Department Technical Report UCLA-CSD TR-01-0023, 2001. 